Louisiana State University

LSU Digital Commons

$2-1-2020$

\title{
Bromodomain and Extraterminal Inhibition by JQ1 Produces Divergent Transcriptional Regulation of Suppressors of Cytokine Signaling Genes in Adipocytes
}

\author{
Paula Mota De Sá \\ Pennington Biomedical Research Center \\ Allison J. Richard \\ Pennington Biomedical Research Center \\ Jacqueline M. Stephens \\ Pennington Biomedical Research Center
}

Follow this and additional works at: https://digitalcommons.Isu.edu/biosci_pubs

\footnotetext{
Recommended Citation

De Sá, P., Richard, A., \& Stephens, J. (2020). Bromodomain and Extraterminal Inhibition by JQ1 Produces Divergent Transcriptional Regulation of Suppressors of Cytokine Signaling Genes in Adipocytes. Endocrinology (United States), 161 (2) https://doi.org/10.1210/endocr/bqz034
} 


\title{
Bromodomain and Extraterminal Inhibition by JQ1 Produces Divergent Transcriptional Regulation of Suppressors of Cytokine Signaling Genes in Adipocytes
}

\author{
Paula Mota de Sá, ${ }^{1,2}$ Allison J. Richard, ${ }^{1}$ and Jacqueline M. Stephens ${ }^{1,2}$ \\ ${ }^{1}$ Adipocyte Biology Laboratory, Pennington Biomedical Research Center, Baton Rouge, Louisiana 70808; \\ and ${ }^{2}$ Department of Biological Sciences, Louisiana State University, Baton Rouge, Louisiana 70803
}

ORCiD numbers: 0000-0002-9559-4061 (A. J. Richard).

\begin{abstract}
The Janus kinase-signal transducer and activator of transcription (JAK-STAT) signaling pathway has cell-specific functions. Suppressors of cytokine signaling (SOCS) proteins are negativefeedback regulators of JAK-STAT signaling. STAT5 plays a significant role in adipocyte development and function, and bromodomain and extraterminal (BET) proteins may be involved in STAT5 transcriptional activity. We treated 3T3-L1 adipocytes with the BET inhibitor JQ1 and observed that growth hormone (GH)-induced expression of 2 STAT5 target genes from the SOCS family, Socs3 and Cish, were inversely regulated (increased and decreased, respectively) by BET inhibition. Chromatin immunoprecipitation analyses revealed that changes in STAT5 binding did not correlate with gene expression changes. GH promoted the recruitment of the BET protein BRD2 to the Cish, but not Socs3, promoter. JQ1 treatment ablated this effect as well as the GHinduced binding of ribonucleic acid polymerase II (RNA Pol II) to the Cish transcription start site. BRD2 knockdown also suppressed GH induction of Cish, further supporting the role of BRD2 in Cish transcriptional activation. In contrast, JQ1 increased the binding of activated Pol II to the Socs3 coding region, suggesting enhanced messenger RNA (mRNA) elongation. Our finding that JQ1 transiently reduced the interaction between the positive transcription elongation factor (P-TEFb) and its inhibitor hexamethylene bis-acetamide inducible 1 (HEXIM1) is consistent with a previously described off-target effect of JQ1, whereby P-TEFb becomes more available to be recruited by genes that do not depend on BET proteins for activating transcription. These results demonstrate substantially different transcriptional regulation of Socs3 and Cish and suggest distinct roles in adipocytes for these 2 closely related proteins. (Endocrinology 161: 1-15, 2020)
\end{abstract}

Key Words: growth hormone, STAT5, adipocytes, BET proteins, P-TEFb, HEXIM1

$\mathbf{S}$ ignal transducers and activators of transcription (STATs) are a family of transcription factors expressed in most cells and tissues. In mammals, there are 7 members in the family (STATs 1, 2, 3, 4, 5A, 5B, and 6), which are activated by different ligands, depending on cell type. Upon external stimulation, STATs dimerize in the cytosol and translocate to the nucleus where they control the expression of target genes (1). The suppressors of cytokine signaling (SOCS) family

ISSN Online 1945-7170

C) Endocrine Society 2019. All rights reserved. For permissions, please e-mail: journals. permissions@oup.com

Received 30 August 2019. Accepted 18 December 2019

First Published Online 26 December 2019.

Corrected and Typeset 8 February 2020. has 8 members, SOCS1 through SOCS7 and cytokineinducible $\mathrm{SH} 2$-containing protein (CISH), all of which are well-established STAT target genes. These proteins are rapidly transcribed and translated following Janus kinase-signal transducer and activator of transcription

\footnotetext{
Abbreviations: ANOVA, analysis of variation; BET, bromodomain and extraterminal; CALF, bovine calf serum; CISH, cytokine-inducible SH2-containing protein; CDNA, complementary DNA; DMEM, Dulbecco's Modified Eagle Media; DMSO, Dimethyl sulfoxide; DNA, deoxyribonucleic acid; FBS, fetal bovine serum; GH, growth hormone; GHR, GH receptor; HEXIM1, hexamethylene bis-acetamide inducible 1; HIV, human immunodeficiency virus; IP, immunoprecipitation; JAK, Janus kinase; KD, knockdown; m, mouse; mRNA, messenger RNA; NT, nontargeting; Pol II, polymerase II; P-TEFb, positive transcription elongation factor; qPCR, quantitative polymerase chain reaction; RNA, ribonucleic acid; RT, reverse transcription; SEC, super elongation complex; SDS, sodium dodecyl sulfate; Ser2, serine 2; siRNA, small interfering RNA; SOCS, suppressors of cytokine signaling; STAT, signal transducer and activator of transcription; TSS, transcription start site.
} 
(JAK-STAT) pathway activation, and they function to turn off JAK-STAT signaling via a negative feedback loop (2). In adipocytes, growth hormone (GH) or prolactin can activate STAT5 $(3,4)$, and a number of studies have demonstrated that this transcription factor is important for adipocyte development and function (5). The regulation of important physiological processes, such as carbohydrate and lipid metabolism, by GH is, at least in part, accomplished by its effects on gene transcription (6). For example, GH can upregulate insulin growth factor 1 in the liver and other tissues, including adipose tissue $(7,8)$. Conversely, $\mathrm{GH}$-induced binding of STAT5 to the promoter of B-cell lymphoma 6 (BCL6) gene inhibits its expression (9).

Bromodomain and extraterminal (BET) proteins (BRDT, BRD2, BRD3, and BRD4) are epigenetic readers that bind to acetylated histones and promote transcription, forming a link between chromatin remodeling and transcription factors. BETs can recruit the positive transcription elongation factor $\mathrm{b}$ (P-TEFb), a cyclindependent kinase composed of 2 cyclin-dependent kinase 9 catalytic subunits and 2 regulatory subunits, which are usually cyclin T1 (10). P-TEFb phosphorylates the C-terminal domain of ribonucleic acid polymerase II (RNA polymerase II) on serine 2 (Ser2), an essential modification for transcription elongation (11). Inhibition of BET proteins by small molecules has been extensively studied for treating cancer (12-14) and inflammatory states $(15,16)$. BET inhibition is generally associated with reduced transcriptional activation and, consequently, with decreased gene expression. Notably, however, studies have shown that gene transcription of human immunodeficiency virus (HIV)-1 is dependent on P-TEFb (17) and can be increased by BET inhibition $(18,19)$. This upregulation is explained by a secondary effect of BET inhibitors to release P-TEFb from its inhibitory complex so that it is more available for transcript elongation $(18,20)$. In normal cellular conditions, where inducible genes contain Pol II poised at their promoters, elongation is tightly regulated by the equilibrium between inactive $\mathrm{P}-\mathrm{TEFb}$ that is associated with the scaffold 7SK noncoding RNA and HEXIM1 (hexamethylene bis-acetamide inducible 1) and a free active form of $\mathrm{P}-\mathrm{TEFb}$ that can be recruited by other proteins including BRD4 and the super elongation complex (SEC) (18). BET proteins have also been shown to be involved in STAT5 transcriptional activity $(14,21)$ and may, therefore, play a role in the regulation of STAT5 target genes in adipocytes.

The goal of our study was to examine how BET inhibition would affect the ability of $\mathrm{GH}$ to regulate STAT5 target genes using murine 3T3-L1 adipocytes as a model system. The effects of the BET inhibitor
JQ1 were opposite for 2 members of the SOCS family. GH-mediated upregulation of Cish was decreased by BET inhibition, while induction of Socs 3 was substantially increased. Chromatin immunoprecipitation analysis demonstrated that changes in STAT5A binding to the promoter region of these genes did not necessarily correlate with changes in gene expression. We observed that the BET protein BRD2 was recruited to the Cish promoter by $\mathrm{GH}$ and that this recruitment was decreased by JQ1. Moreover, BRD2 knockdown (KD) decreased GH induction of Cish, further supporting the dependency of Cish transcriptional activation on this BET protein. In contrast, the upregulation of Socs 3 by JQ1 was attributed to enhanced mRNA elongation. BET inhibitors have been reported to release $\mathrm{P}-\mathrm{TEFb}$ from its inhibitory complex and upregulate HEXIM1 (18). In our study, the BET inhibitor JQ1 transiently decreased the interaction between the regulatory subunit of P-TEFb (Cyclin T1) and HEXIM1, rendering more of the elongation factor available for recruitment to the Socs 3 promoter. Our data underscore the complexity of the transcriptional effects of epigenetic modulators and suggest that Socs 3 and Cish may have different functions in adipocytes.

\section{Experimental Procedures}

\section{Cell culture}

Murine 3T3-L1 preadipocytes (originally obtained from lab H, Green lab at Harvard Medical School) were grown to 2 days after confluence in Dulbecco's Modified Eagle Media (DMEM; Sigma-Aldrich, St. Louis, MO) with $10 \%$ bovine calf serum (CALF). Cells were induced to differentiate using a standard MDI induction cocktail of $0.5 \mathrm{mM} 3$-isobutyl-1methylxanthine (MIX), $1 \mu \mathrm{M}$ dexamethasone, and $1.7 \mu \mathrm{M}$ insulin in DMEM containing $10 \%$ characterized fetal bovine serum (FBS). Cell identity was confirmed for each group of cells by monitoring their ability to differentiate into adipocytes, which contain lipid droplets that can be observed via phase-contrast microscopy. HyClone CALF and FBS were purchased from Thermo Scientific or GE Healthcare Life Sciences (Logan, UT). The medium was changed every 48 to 72 hours during all phases of growth and differentiation. When specified, cells were serum deprived with DMEM containing $0.3 \%$ bovine serum albumin (BSA; Cat \#: A6003, Sigma-Aldrich, St. Louis, MO). Otherwise, cells were serum deprived by changing the media to DMEM containing 1\% CALF for 16 to 24 hours before treatment with the inhibitor. (+)-JQ1 was purchased from Sigma-Aldrich (Millipore Sigma). 
Recombinant murine GH was purchased from Dr A.F. Parlow at the National Hormone and Peptide Program (NHPP; Torrance, CA).

\section{Antibodies}

Antibodies against BRD2 (22) (D89B4; rabbit monoclonal), lamin B1 (23) (D4Q4Z; rabbit monoclonal), and rabbit IgG (24) (2729; rabbit polyclonal) were purchased from Cell Signaling Technology (Danvers, MA). Anti-RNA Pol II CTD repeat YSPTSPS (pS2) (25) (ChIP grade ab5095; rabbit polyclonal), cyclin T1 (26) (ab184703; rabbit monoclonal), HEXIM1 (27) (ab25388; rabbit polyclonal), STAT5A (28) (E289; rabbit monoclonal; for WB Cat\# ab32043; for ChIP Cat\#: ab213219) were purchased from Abcam (Cambridge, MA). Anti-BRD4 (29) (PA5-41550; rabbit polyclonal) was purchased from ThermoFisher Scientific (Waltham, MA). Anti-histone H3 (30) (06599; rabbit polyclonal), histone H4 (31) (06-866; rabbit polyclonal), RNA Pol II (32) (17-620; mouse monoclonal) and control mouse IgG were purchased from Millipore Sigma (Burlington, MA).

\section{Reverse transcription-quantitative polymerase chain reaction}

Total RNA was purified from harvested 3T3-L1 adipocyte monolayers using the RNeasy mini kit from Qiagen (Hilden, Germany). RNA levels were quantified by spectrophotometry. Complementary deoxyribonucleic acid (cDNA) was synthesized by reverse transcription (RT) with the High-Capacity cDNA Reverse Transcription kit (Applied Biosystems, Foster City, CA), according to the manufacturer's protocol. Genes of interest were quantified by real-time quantitative PCR (qPCR) using Takara SYBR Premix (Takara Bio USA, Mountain View, CA) and the Applied Biosystems 7900HT Fast Real-Time PCR System. Cyclophilin a and NoNo were used as reference genes. The following mouse $(\mathrm{m})$ genes were examined by RT-qPCR: $m$ Cyclophilin a, mNoNo, mSocs3, $m$ Cish, mBcl6, mIgf1, mSocs 2, mBrd2, mHexim1 using primers purchased from Integrated DNA Technologies (IDT, Skokie, IL). Primer sequences for each gene are shown in Table 1.

\section{Chromatin immunoprecipitation}

The medium on mature 3T3-L1 adipocytes (7-15 days post-MDI) was replaced with $1 \%$ CALF for 16 to 24 hours. Following a 30-minute pretreatment with JQ1 or dimethyl sulfoxide (DMSO) (control), cells were treated with vehicle or $\mathrm{GH}$ for 30 minutes. DNA-protein crosslinking, nuclear preparation, chromatin digestion, and chromatin immunoprecipitation (ChIP) assays were performed using the SimpleChIP kit (Magnetic Beads) from Cell Signaling Technology (Cat \#: 9003S; Danvers, MA) according to the manufacturer's instructions. For each ChIP, 8 to $12 \mu \mathrm{g}$ of digested, cross-linked chromatin were incubated with the following amounts of antibodies, as recommended by their respective manufacturers: rabbit anti-STAT5A (28) $(2 \mu \mathrm{g})$, mouse antiRNA Pol II (32) (1 $\mu \mathrm{g})$, rabbit anti-phosphoserine2 RNA Pol II (25) $(4 \mu \mathrm{g})$, rabbit anti-BRD2 (22) 1:50 dilution, rabbit BRD4 (29) $(1 \mu \mathrm{g})$, rabbit ac-histone $\mathrm{H} 3(5 \mu \mathrm{L})$, rabbit ac-histone $\mathrm{H} 4(5 \mu \mathrm{L})$, nonimmune IgG control antibody rabbit $\operatorname{IgG}(24)$ from ChIP kit $(2 \mu \mathrm{g})$ or mouse IgG from RNA Pol II kit $(1 \mu \mathrm{g})$. After protein digestion and DNA purification using the columns provided in the SimpleChIP kit, input and immunoprecipitated DNA samples were analyzed using real-time qPCR on the Applied Biosystems 7900HT Fast Real-Time PCR System. We used the qPCR amplification program specified in the kit instructions with the exception that the initial denaturation step was performed for 10 minutes at $95^{\circ} \mathrm{C}$. We also added a dissociation curve step at the end of the reaction. Primers used to amplify different regions of the Cish and Socs 3 genes are described in Table 2 . A standard curve prepared with only $2 \%$ input samples and 5-fold serial dilutions was made to assess amplification efficiency.

IP efficiency was calculated using the percent input approach and the following equation: Percent

\section{Table 1. Primers Used for RT-qPCR}

\begin{tabular}{lll}
\hline Gene & Primer $\mathbf{1}$ & Primer $\mathbf{2}$ \\
\hline Bcl6 & 5'-AGTCACATTCGTTGCAGAAGA-3' & 5'-CAGAGATGTGCCTCCATACTG-3' \\
Brd2 & 5'-GGTTCCCTGAGGTCAAGATG-3' & 5'-TCGAATCCTTTTCCCTGGTG-3' \\
Cish & 5'-CCGCCCAATTTGCTCCA-3' & 5'-GCTCCTTCTCCTTCCATCC-3' \\
Cyclophilin a (Ppia) & 5'-TCTTCAGGTCAATAGTCAAGCC-3' & 5'-TGCAAACAGCTCGAAGGAGACGC-3' \\
Hexim1 & 5'-CAGCCTCAAACTAGCAACTGTA-3' & 5'-CTCGATTGCCACCTACTGTC-3' \\
lgf1 & 5'-ATGCTCTTCAGTTCGTGTGT-3' & 5'-AGTACATCTCCAGTCTCCTCAG-3' \\
Nono & 5'-CATCATCAGCATCACCACCA-3' & 5'-TCTTCAGGTCAATAGTCAAGCC-3' \\
Socs2 & 5'-TCTGGGGACTGCCTTTACCAAC-3' & 5'-CCTCTGGGTTCTCTTTCACATAGC-3' \\
Socs3 & 5'-GGAAACTTGCTGTGGGTGA-3' & 5'-GAGATTTCGCTTCGGGACTA-3' \\
\hline
\end{tabular}

Abbreviation: RT-qPCR, reverse transcription-polymerase chain reaction. 


\section{Table 2. Primers Used for ChIP-qPCR}

\begin{tabular}{lll}
\hline Gene Region & Forward & Reverse \\
\hline Cish SBS & 5'-CGTCCAGCGATACGATTGGT-3' & 5'-CAGGCGTCTAGTGCTTTGGA-3' \\
Cish TSS & 5'-GTTCGCACCACAGCCTTTCAGTCC-3' & 5'-TGCCAGGGGTGCGAAGGTCAGG-3' \\
Cish Coding & 5'-TACCCCTTCCAACTCTGACTGAGC-3' & 5'-TTCCCTCCAGGATGTGACTGTG-3' \\
Socs3 SBS & 5'-GCACAGCCTTTCAGTGCAGAG-3' & 5'-GTATTTACCCGGCCAGTACGC-3' \\
Socs3 TSS & 5'-GCGTACTGGCCGGGTAAATAC-3' & 5'-GGAGAGACAGCGGTCGTAAG-3' \\
Socs3 Coding & 5'-CATTTAGAAGGGAGACAGATGAG-3' & 5'-CATAGGAGAGACAAAGCAGAAC-3' \\
\hline
\end{tabular}

Abbreviation: ChIP, chromatin immunoprecipitation; qPCR, quantitative polymerase chain reaction; SBS, STAT binding site; TSS, transcription start site.

\section{Socs 3}

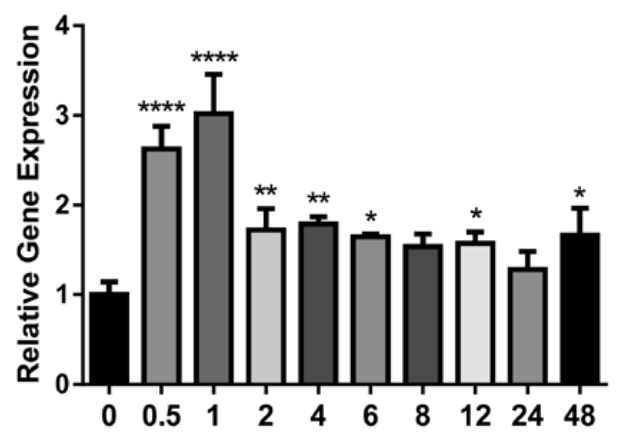

lgf1

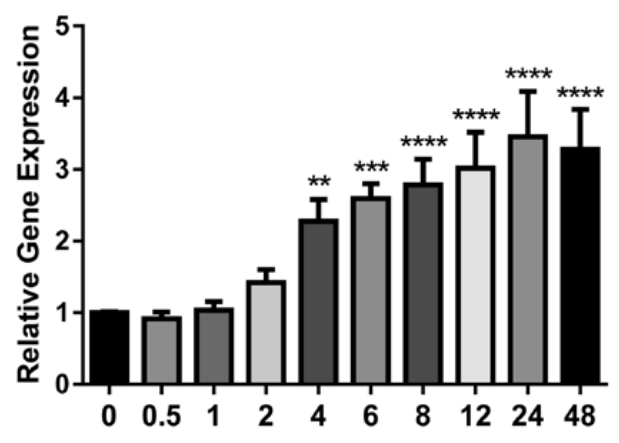

Cish

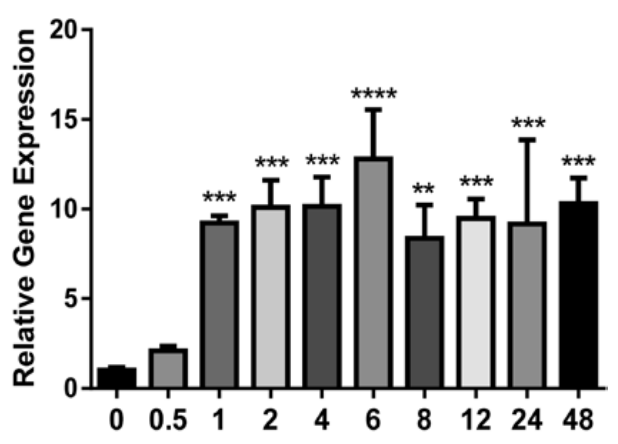

Socs2

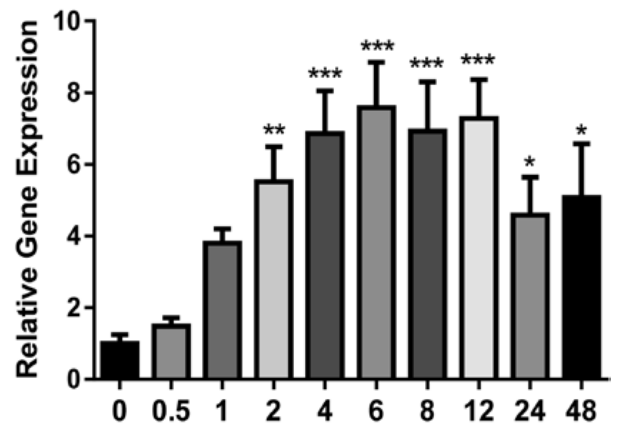

$B c / 6$

Figure 1. Regulation of STAT5 target genes by GH. Mature 3T3-L1 adipocytes were treated with $5 \mathrm{nM}$ GH in triplicate and harvested at indicated time points. Relative gene expression of Socs3, Cish, lgf1, Socs2, and Bcl6 were quantified by RT-qPCR using cyclophilin a as the housekeeping gene. This experiment was replicated at least 3 times in different batches of cells. Data are presented as means $\pm S E(n=3$ per treatment). All time points are compared to time 0 . Statistical significance was determined using one-way ANOVA. ${ }^{*} P<0.05, * * P<0.005, * * * P<0.001$, $* * * * P<0.0001$. Abbreviations: ANOVA, analysis of variance; GH, growth hormone; RT-qPCR, reverse transcription-quantitative polymerase chain reaction; SE, standard error; STAT, signal transducer and activator of transcription.

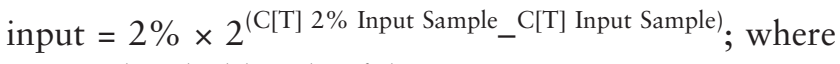
$\mathrm{C}[\mathrm{T}]=$ threshold cycle of the $\mathrm{PCR}$ reaction. Percent input of control rabbit and mouse IgG was calculated for all the regions analyzed and ranged from $0.03 \%$ to $0.1 \%$. The coding regions of Socs 3 and Cish were used as control regions lacking STAT binding sites. STAT5-specific 
binding was at least 50-fold higher at Cish promoter and 30-fold higher at Socs3 promoter when compared with the nonspecific coding region or for nonimmune IgG binding at the same sites. BRD2 binding to Cish promoter was 10 -fold higher than binding to the coding region, while BRD2 binding to Socs3 promoter was only 2 -fold higher than binding to the coding region.

\section{Preparation of whole-cell extracts}

Cell monolayers were rinsed once with phosphate buffered saline and then scraped into nondenaturing IP buffer that contained $10 \mathrm{mM}$ Tris ( $\mathrm{pH} 7.4$ ), $150 \mathrm{mM} \mathrm{NaCl}$, $1 \mathrm{mM}$ ethylene glycol-bis( $\beta$-aminoethyl ether)-N,N,N',N'tetraacetic acid (EGTA), $1 \mathrm{mM}$ ethylenediaminetetraacetic acid (EDTA), 1\% Triton X-100, 0.5\% IGEPAL CA-630, protease inhibitors (1 $\mathrm{mM}$ phenylmethylsulfonyl fluoride [PMSF], $1 \mu \mathrm{M}$ pepstatin, 50 trypsin inhibitory milliunits of aprotinin, $10 \mu \mathrm{M}$ leupeptin, $1 \mathrm{mM} 1$, 10-phenanthroline), and phosphatase inhibitors $(100 \mu \mathrm{M}$ sodium fluoride and $0.2 \mathrm{mM}$ sodium vanadate). The cell suspension was subjected to a freeze/thaw cycle at $-80^{\circ} \mathrm{C}$ and then passed through a 20-gauge needle 5 times. The whole-cell extract was clarified by centrifugation at $13000 \times \mathrm{g}$ for 10 minutes at $4^{\circ} \mathrm{C}$.

\section{Measurement of protein concentration}

Protein content of cell and tissue extracts was determined using the bicinchoninic acid assay kit from Sigma-Aldrich (Cat \#: BCA1).

\section{Gel electrophoresis and immunoblotting}

Samples were separated on $6 \%$ or $7.5 \%$ sodium dodecyl sulfate (SDS) polyacrylamide gels (acrylamide, Cat \#: EC-890, from National Diagnostics, Atlanta, GA) and transferred to nitrocellulose membranes (Cat \#: 162-0115, BioRad, Hercules, CA). Results were visualized with horseradish peroxidase-conjugated secondary antibodies (Jackson ImmunoResearch, West Grove, PA) and enhanced chemiluminescence (Pierce/ Thermo Scientific, Rockford, IL).

\section{Small interfering RNA-mediated knockdown}

3T3-L1 mature adipocytes (5-7 days post-MDI) were trypsinized from 6-well plates and re-plated in 12-well plates at a density of $1.16 \times 10^{5}$ cells $/ \mathrm{cm}^{2}$ in antibioticfree medium (10\% FBS/DMEM). Adipocytes were transfected using DharmaFECT Duo reagent (Dharmacon; Cat \#: T-2010-03) and $50 \mathrm{nM}$ of nontargeting (NT; Cat \#: D-001810-10-50) or Brd2 (Cat \#: L-04340400-0005) small interfering RNA (siRNA) in OptiMEM reduced serum medium (Thermo Fisher; Cat \#: 31985088). Twenty-four hours later, siRNA-containing media was removed and replaced with antibiotic-free
10\% FBS/DMEM. After 24 hours, adipocytes were transfected again with NT or Brd2 siRNA. siRNAcontaining medium was removed 24 hours later and replaced with $10 \%$ FBS. After 24 hours, FBS was replaced by $1 \%$ CALF, antibiotic-free. The next day, cells were treated with vehicle or $5 \mathrm{nM} \mathrm{GH}$ for 1 hour. Adipocytes were harvested in RLT buffer (Qiagen) for RNA analysis, and whole-cell extracts were collected in IP buffer for protein quantification.

\section{Immunoprecipitation}

Cell extracts (300 $\mu \mathrm{g}$ total protein) were incubated with $5 \mu$ l of anti-cyclin T1 antibody overnight on a mini-tube rotator at $4^{\circ} \mathrm{C}$. Protein A-conjugated agarose (IPA300 Protein A Resin; Cat \#: 10-2003-02) from Repligen (Waltham, MA) or Protein A/G Plus-agarose (Cat \#: sc-2003) beads from Santa Cruz Biotechnology (Dallas, TX) were added to the antibody-epitope mixture. The conjugation reaction continued for an additional 1 to 2 hours at $4^{\circ} \mathrm{C}$ with rotation. Next, the beads were pelleted by centrifugation at $13000 \mathrm{rpm}$ for 3 minutes at $4^{\circ} \mathrm{C}$. The supernatant was removed by aspiration, and the beads were washed 3 times with ice-cold 1X IP buffer containing protease inhibitors. Between each wash, the beads were pelleted by centrifugation at $12000 \mathrm{rpm}$ for 1 minute at $4^{\circ} \mathrm{C}$ and the supernatant removed by aspiration. After the final wash, the IP antibody and immunoprecipitated proteins were eluted from the bead resin into 2X SDS loading buffer by boiling the samples for 10 minutes at $100^{\circ} \mathrm{C}$. Samples were flicked multiple times during the heat step to ensure efficient elution. The samples were briefly centrifuged and the supernatants analyzed by sodium dodecyl sulfate-polyacrylamide gel electrophoresis (SDS-PAGE) and immunoblotting. A mock sample containing a pool of equal concentration of all samples and no IP antibody was used as a negative control to ensure that there was no unspecific binding of the beads to protein samples.

\section{Statistical analysis}

Data were plotted as mean \pm standard error (SE). The qPCR data were analyzed using either using one-way analysis of variation (ANOVA) or two-way ANOVA. For ChIP experiments, data were analyzed using two-way ANOVA with the Bonferroni post hoc test. Differences with $P<0.05$ were considered statistically significant.

\section{Results}

\section{GH regulation of STAT5 target genes in adipocytes}

In order to study the modulation of STAT5 target genes in 3T3-L1 adipocytes, cells were treated with GH 
and harvested at different time points, up to 48 hours. The expression of STAT5 target genes was analyzed by RT-qPCR (Fig. 1). As expected, Socs 3 and Cish were acutely regulated by $\mathrm{GH}$ treatment. The induction of Socs 3 mRNA was evident at 30 minutes, and the maximal response was observed at one hour. Cish was upregulated at one hour and remained elevated after 48 hours. Bcl6 expression was downregulated by $\mathrm{GH}$ at early time points and remained low over the course of treatment. Socs 2 and Igf1 were also induced by GH, but at later time points. Since Socs 3 and Cish are well characterized in other model systems (33-37) and were acutely induced by GH at early time points, we focused our additional studies on these 2 STAT5 target genes.

\section{BET inhibition increased GH-induced Socs3 expression and decreased $\mathrm{GH}$-induced Cish expression}

The thienotriazolodiazepine JQ1 competitively binds to bromodomains of BET proteins and prevents their binding to chromatin. Only (+)-JQ1 interacts with bromodomains; the enantiomer (-)-JQ1 does not inhibit BET proteins (38). As shown in Fig. 2A, BET inhibition

A.

Cish

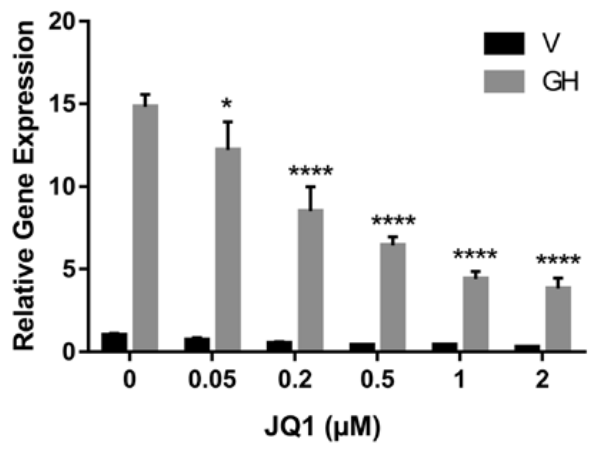

B.

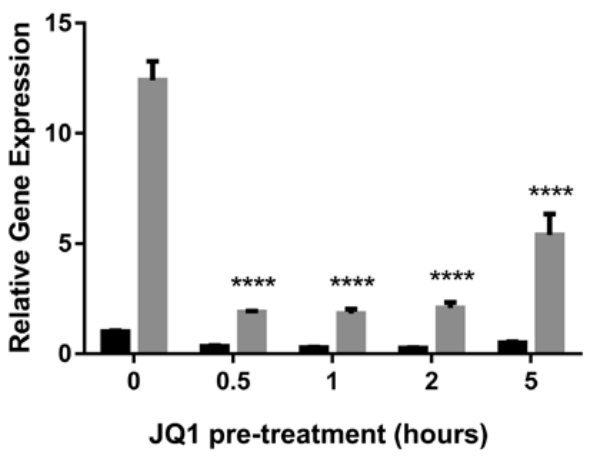

using the active enantiomer (+)-JQ1 decreased GH induction of Cish and increased Socs 3 upregulation in a dose-dependent manner. In addition, the effects of JQ1 on GH induction of Cish and Socs 3 were acute, as only 30 minutes of pretreatment were sufficient to produce these opposite effects on Cish and Socs 3 expression (Fig. 2B). The effects of BET inhibition decreased over time, probably because of JQ1's short half-life (39). In vehicle conditions, there were no statistically significant differences between treatments.

\section{GH recruits BRD2 to the Cish promoter, and JQ1 decreases BRD2 and RNA Pol II recruitment}

Since GH treatment induces the JAK-STAT signaling pathway and STATs modulate transcription of target genes, we investigated the effects of JQ1 on binding of various transcriptional regulators to distinct sites in the promoters or coding sequences of the Socs3 or Cish genes. Fig. 3A illustrates the 3 target regions of Cish that were amplified by qPCR: one region containing STAT binding sites (SBS), another containing the transcription start site (TSS), and a third within the coding region of Cish. Results demonstrated that GH and JQ1 each modestly increased STAT5A binding to the Cish
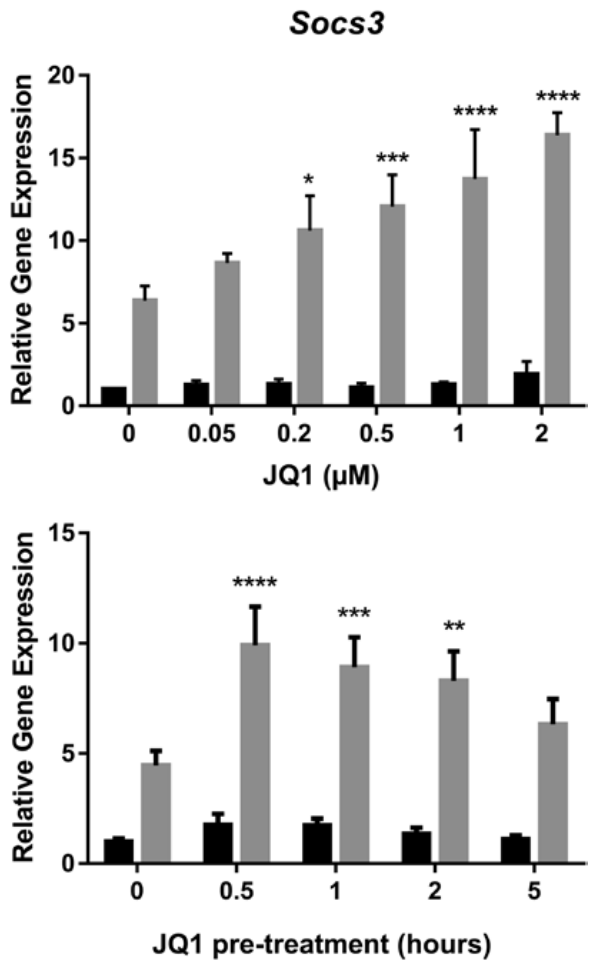

Figure 2. JQ1 upregulates Socs3 and downregulates Cish. (A) Mature 3T3-L1 adipocytes were pretreated for 30 minutes with JQ1 at different concentrations. Vehicle or $5 \mathrm{nM}$ of GH were added 1 hour before the cells were harvested. (B) Mature adipocytes were pretreated with $1 \mu \mathrm{M}$ JQ1 for indicated times, and vehicle or $5 \mathrm{nM}$ of GH were added 1 hour before the cells were harvested. Gene expression levels were quantified by RT-qPCR. Cyclophilin a was used as the reference gene. Data are presented as means \pm SE ( $n=3$ per treatment). This experiment was replicated 3 times in different batches of cells. Treatments were normalized to vehicle condition in the absence of inhibitor. Statistical significance was determined using two-way ANOVA. ${ }^{*} P<0.05, * * P<0.005, * * * P<0.001, * * * * P<0.0001$. Abbreviations: ANOVA, analysis of variance; GH, growth hormone; RT-qPCR, reverse transcription-quantitative polymerase chain reaction; SE, standard error. 
A.

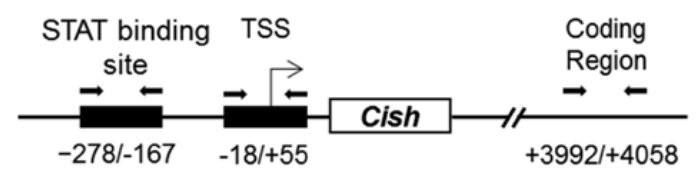

C.

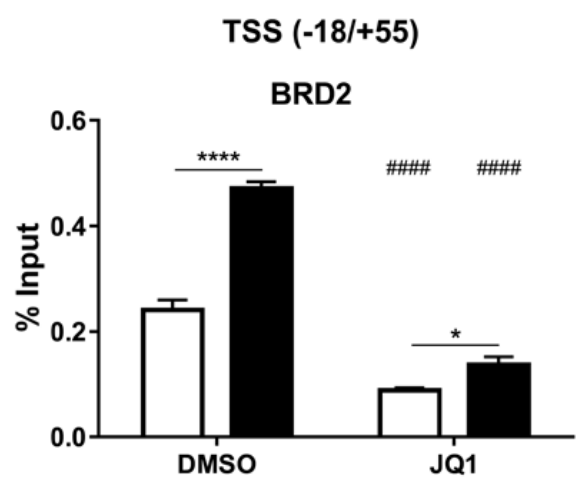

E.

TSS (-18/+55)

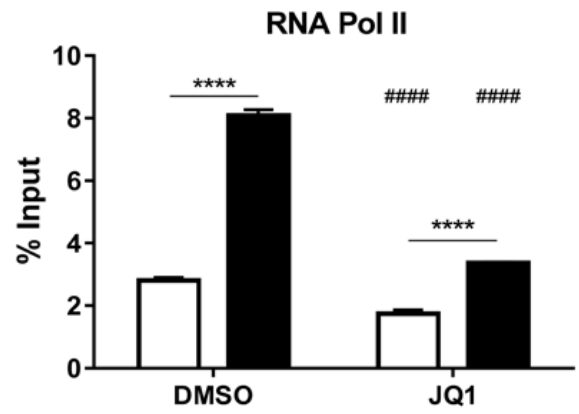

G.

TSS (-18/+55)

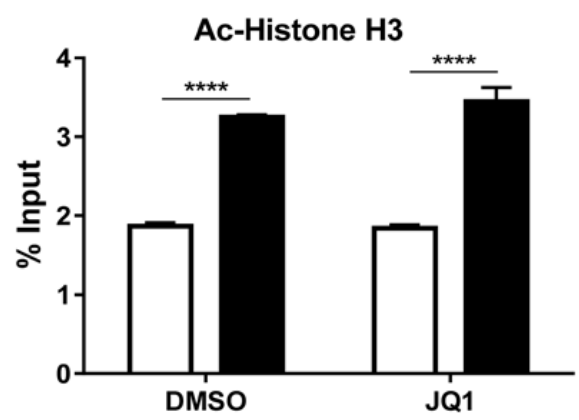

B.

STAT Binding Site (-278/-167)

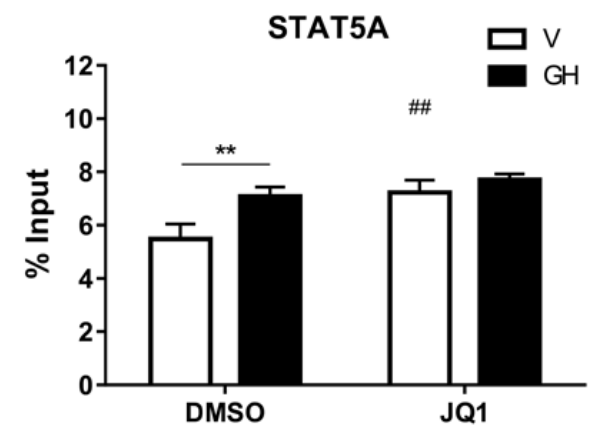

D.

TSS (-18/+55)

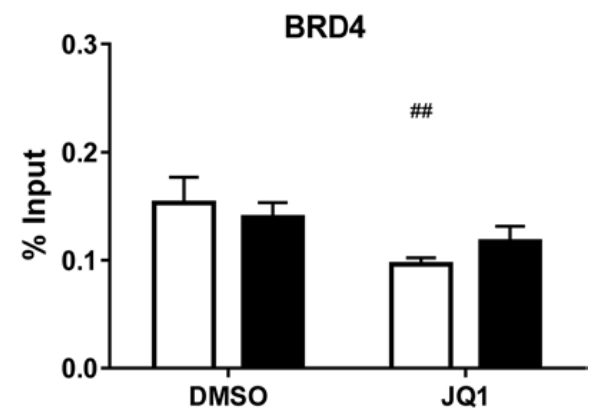

F.

Coding Region (+3992/+4058)

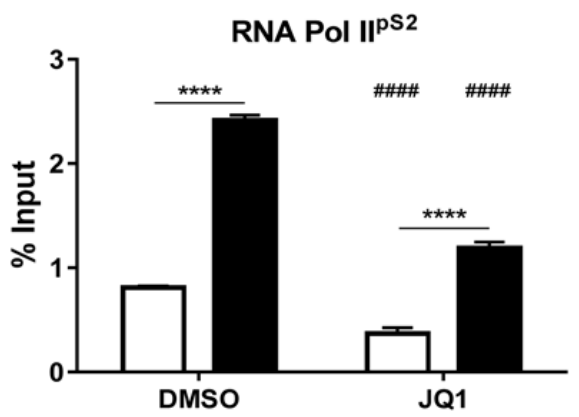

$\mathrm{H}$.

TSS (-18/+55)

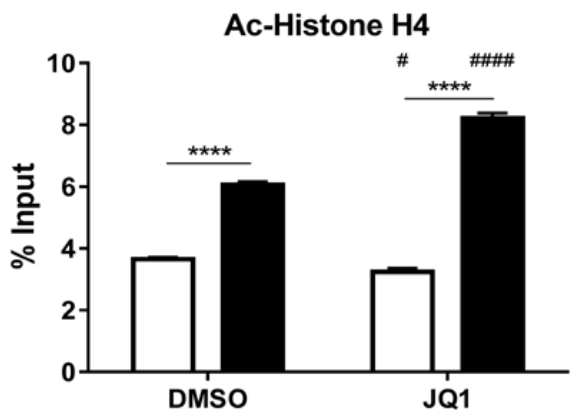

Figure 3. Recruitment of RNA Pol II to Cish TSS highly correlates to BRD2 recruitment. (A) Schematics of Cish regions that were amplified by qPCR in ChIP assay, including STAT binding site, transcription start site (TSS) and coding region of the gene. (B-F). Mature adipocytes were pretreated with $2 \mu \mathrm{M}$ JQ1 or DMSO for 30 minutes. Next, cells were treated with vehicle $\left(\mathrm{V} ; \mathrm{NaHCO}_{3}\right)$ or $5 \mathrm{nM} \mathrm{GH}$ for 35 minutes. Chromatin was cross-linked using formaldehyde, and nuclear extracts were subjected to ChIP using specific antibodies. The amount of bound DNA was measured by qPCR and percentage input was calculated. IP efficiency (\% input) of (B) STAT5A binding to STAT binding site, (C) BRD2, (D) BRD4, (E) total RNA polymerase II, (G) ac-histone H3, (H) ac-histone H4 binding to Cish TSS and (F) RNA Pol II ${ }^{\text {pS2 }}$ binding to Cish coding region. This experiment was replicated 3 times in different batches of cells. Statistical significance was calculated using two-way ANOVA and the Bonferroni post hoc test. * denotes significance among $\mathrm{V}$ and $\mathrm{GH}$ in the presence or absence of JQ1. \# denotes significance between DMSO and JQ1 in the presence or absence

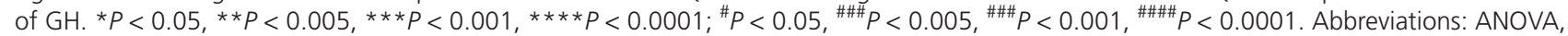
analysis of variance; ChIP, chromatin immunoprecipitation; DMSO, dimethyl sulfoxide; GH, growth hormone; Pol II, polymerase II, RNA, ribonucleic acid; RT-qPCR, reverse transcription-quantitative polymerase chain reaction; STAT, signal transducer and activator of transcription; TSS, transcription start site. 
STAT binding site (Fig. 3B), but that JQ1 had no further effect on STAT5A binding in the presence of GH. As BRD4 is the most well-studied BET protein and BRD2 was previously described to be involved in STAT5 transcriptional regulation $(14,21)$, binding of BRD2 and BRD4 to the Cish TSS was also investigated. BRD2 was recruited to Cish TSS by GH, and its binding was suppressed by JQ1 (Fig. 3C). BRD4 binding levels were low, ranging from $0.06 \%$ to $0.2 \%$ input, only slightly higher than IgG levels ranging from $0.03 \%$ to $0.1 \%$ input (data not shown). In addition, BRD4 binding was not substantially altered by GH or JQ1 treatment (Fig. 3D). As expected, total RNA Pol II binding to Cish TSS was increased by GH treatment. In the presence of JQ1, this RNA Pol II recruitment to Cish TSS was decreased (Fig. 3E). Similarly, the binding of Pol II phosphorylated at Ser2, a marker of RNA elongation, to the coding region of Cish was increased with $\mathrm{GH}$ and suppressed by JQ1 (Fig. 3F). Since BET proteins bind to acetylated histones, we also assessed the levels of total acetylated histone $\mathrm{H} 3$ (Fig. 3G) and $\mathrm{H} 4$ (Fig. 3H) at the Cish TSS. In the presence or absence of JQ1, GH highly increased ac-histone $\mathrm{H} 3$ and $\mathrm{H} 4$ at the Cish promoter. JQ1 further increased only ac-histone H4 in the presence of GH.

\section{JQ1 increases Socs3 mRNA elongation}

Fig. 4A illustrates the 3 target regions of Socs 3 that were amplified by qPCR. STAT5A interaction with its binding site in Socs 3 was strongly enhanced by GH but minimally affected by JQ1 treatment, indicating that the effect of JQ1 on GH-induced Socs3 expression was not the result of increased STAT5A binding (Fig. 4B). In contrast to what we observed at the Cish TSS, BRD2 was not recruited to the Socs 3 TSS by GH, and JQ1 modestly decreased its binding (Fig. 4C). BRD4 binding to the Socs 3 TSS was somewhat higher than binding to the Cish TSS, but it was not substantially affected by GH or JQ1 (Fig. 4D). RNA Pol II recruitment to the Socs3 TSS was increased by GH; JQ1 enhanced Pol II recruitment in the vehicle condition but had no effect in GH conditions (Fig. 4E). Therefore, changes in BRD2 or RNA Pol II recruitment to the $S o c s 3$ promoter did not explain the greater upregulation of Socs3 expression by JQ1 either. Binding of activated Pol II to the Socs 3 coding region, however, was robustly increased by JQ1, suggesting that the upregulation of Socs 3 gene expression by JQ1 is due to augmented mRNA elongation (Fig. 4F). Acetylated histone H3 (Fig. 4G) and H4 (Fig. 4H) levels were much lower at Socs3 TSS when compared to Cish. In addition, neither GH nor JQ1 had any notable influence on acetylated histone $\mathrm{H} 3$ levels. $\mathrm{GH}$ increased ac-histone H4 only in the presence of JQ1.

\section{BRD2 knockdown decreases GH activation of Cish}

Our ChIP experiments demonstrated that BRD2 was recruited to the Cish promoter by GH, and JQ1 decreased this recruitment. To determine whether the effects of JQ1 were dependent on BRD2, we used an siRNA KD approach. Fig. 5A shows mRNA levels of Brd2, Socs3, and Cish. KD efficiency at the mRNA level was about $50 \%$. In basal conditions, neither Cish nor Socs 3 expression was affected by Brd2 KD. However, only GH activation of Cish was reduced by $\mathrm{Brd} 2 \mathrm{KD}$, further supporting the hypothesis that BRD2 is necessary for $\mathrm{GH}$ activation of Cish. In contrast, the upregulation of $S o c s 3$ by JQ1 was not recapitulated by BRD2 KD, ruling out the possibility that BRD2 was acting as a negative regulator of Socs3. BRD2 protein levels were decreased by about $80 \%$ in the KD conditions versus the NT control, and GH had no effect on BRD2 protein expression (Fig. 5B).

\section{JQ1 interferes with P-TEFb availability and upregulates HEXIM1}

To explain why JQ1 upregulates Socs3, we scoured the literature for examples of genes upregulated by BET inhibition. Curiously, JQ1 was reported to upregulate HIV-1 expression through the transient release of $\mathrm{P}-\mathrm{TEFb}$ from its inhibitory complex, leading to compensatory upregulation of HEXIM1 (18). To investigate if this could occur for GH-induced Socs 3 transcription, we treated 3T3-L1 adipocytes with DMSO or JQ1 for 2, 6, or 24 hours and examined Hexim1, Socs3, and Cish gene expression. Interestingly, JQ1 substantially upregulated Hexim1; levels peaked at 6 hours and started to decrease at 24 hours but remained significantly higher than in the DMSO control. Adipocytes were also treated with GH for 1 hour before harvesting for induction of Socs 3 and Cish and for confirmation of inhibitor effects (Fig. 6A). In addition, we measured HEXIM1 protein levels at the same time points and observed a progressive accumulation of HEXIM1 when the cells were treated with JQ1. We also found that JQ1 upregulated BRD2 protein levels. Quantification and fold change of BRD2 and HEXIM1 using cyclin T1 as a control is shown below the Western blot images (Fig. 6B). Lastly, cell lysates were immunoprecipitated with cyclin $\mathrm{T} 1$ antibody (the regulatory portion of $\mathrm{P}-\mathrm{TEFb}$ ) to evaluate the impacts of JQ1 on P-TEFb availability. In Fig. 6C, quantification values of BRD2 and HEXIM1 were divided by cyclin T1, and graphs are shown below 
A.

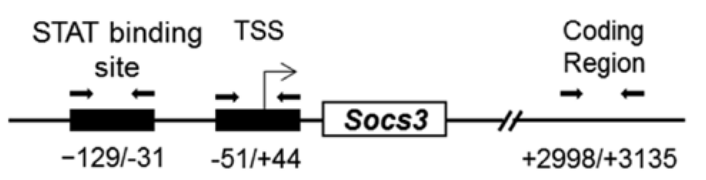

C.

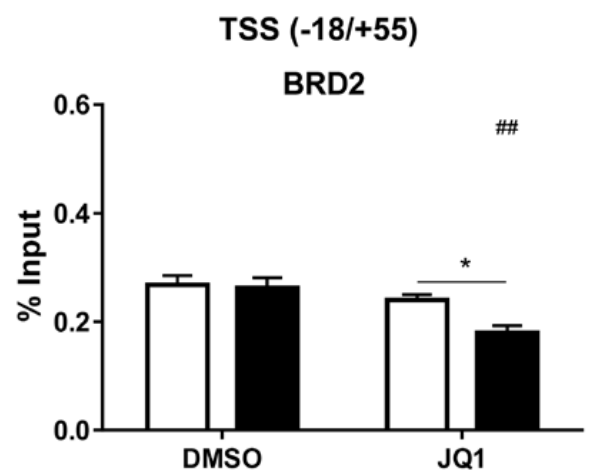

E.

TSS (-18/+55)

RNA Pol II

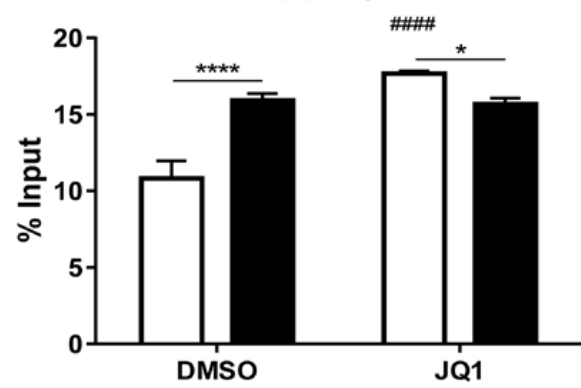

G.

TSS (-18/+55)

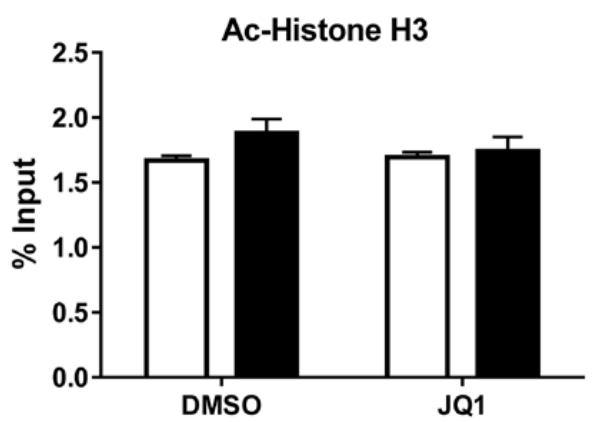

B.

STAT Binding Site (-278/-167)

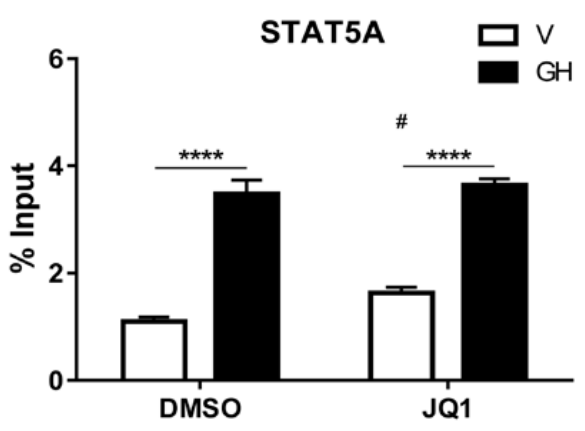

D.

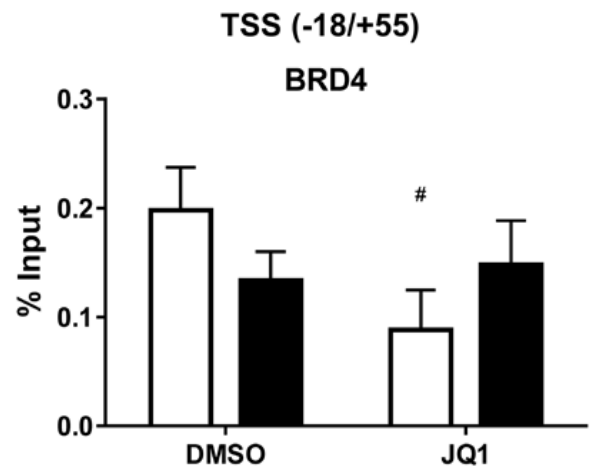

F.

Coding Region (+3992/+4058)

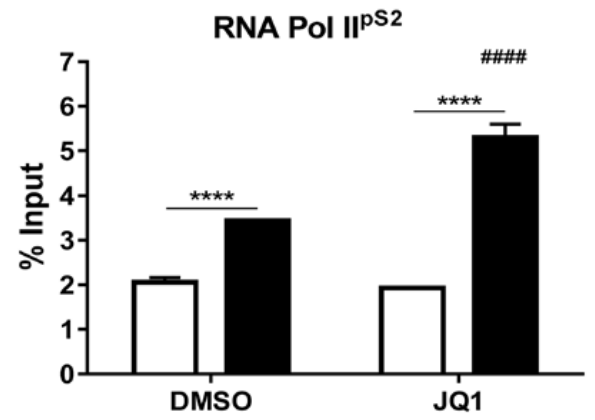

H.

TSS (-18/+55)

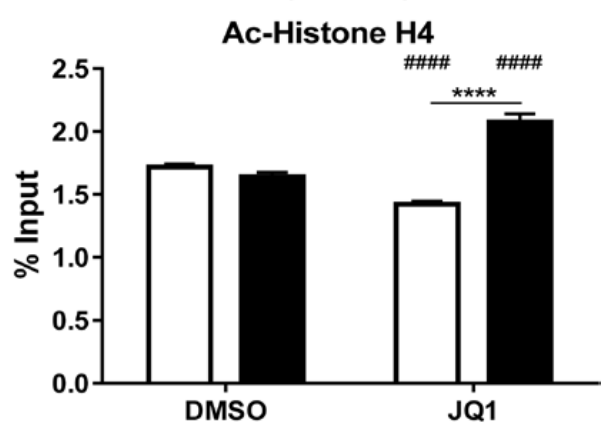

Figure 4. Socs3 mRNA elongation is enhanced by JQ1. (A) Schematics of Socs3 regions that were amplified by qPCR in ChIP assay, including STAT binding site, transcription start site (TSS), and coding region of the gene. (B-F). Mature adipocytes were pretreated with $2 \mu \mathrm{M}$ JQ1 or DMSO for 30 minutes. Next, cells were treated with vehicle $\left(\mathrm{V} ; \mathrm{NaHCO}_{3}\right)$ or $5 \mathrm{nM} \mathrm{GH}$ for 35 minutes. Chromatin was cross-linked using formaldehyde, and nuclear extracts were subjected to ChIP using specific antibodies. The amount of bound DNA was measured by qPCR and percentage input was calculated. IP efficiency (\% input) of (B) STAT5A binding to STAT binding site, (C) BRD2, (D) BRD4, (E) total RNA polymerase II (Pol II), (G) ac-histone H3, (H) ac-histone H4 binding to Cish TSS, and (F) RNA Pol IIS2 binding to Cish coding region. This experiment was replicated 3 times in different batches of cells. Statistical significance was calculated using two-way ANOVA and the Bonferroni post hoc test. * denotes significance among $\mathrm{V}$ and GH in the presence or absence of JQ1. \# denotes significance between DMSO and JQ1 in the presence or absence of

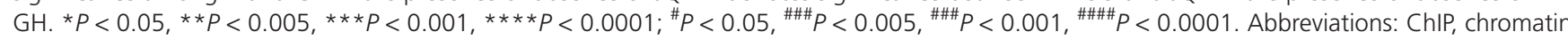
immunoprecipitation; DMSO, dimethyl sulfoxide; DNA, deoxyribonucleic acid; $\mathrm{mRNA}$, messenger ribonucleic acid; qPCR, quantitative polymerase chain reaction; STAT, signal transducer and activator of transcription. 
A.

\section{Brd2}

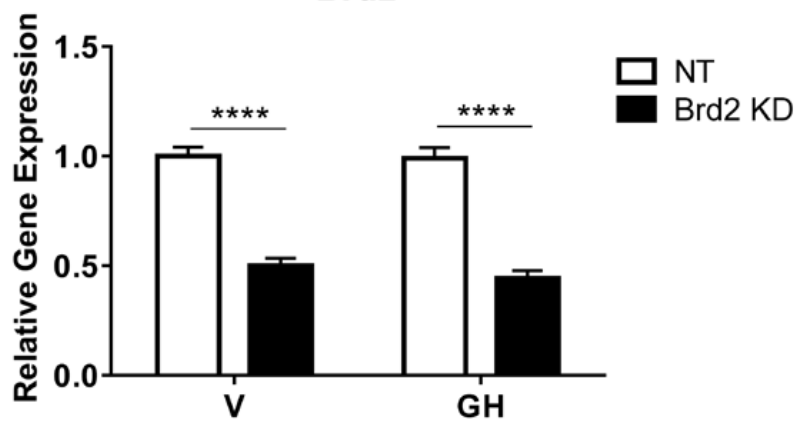

\section{Cish}

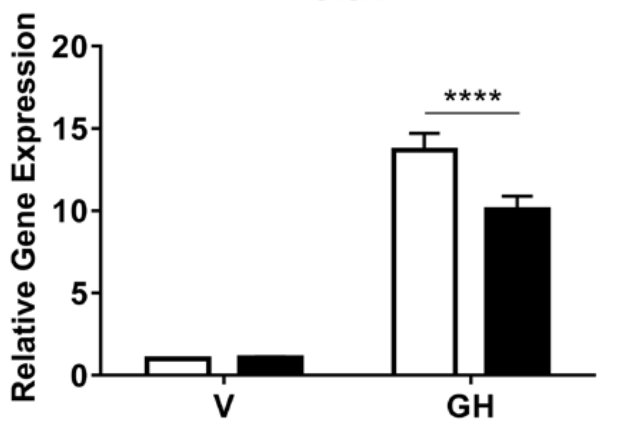

B.
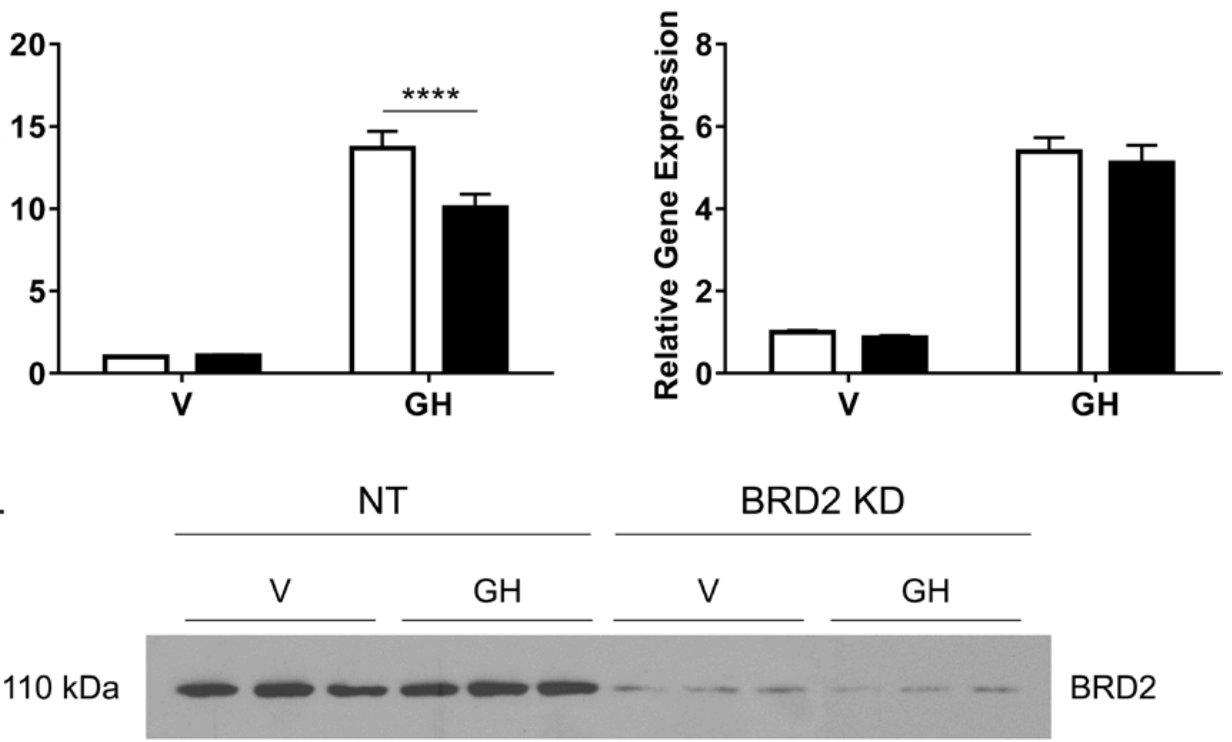

BRD2 KD

BRD2

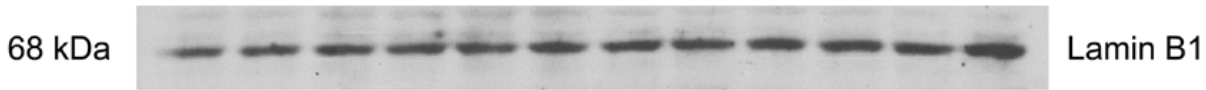

\section{BRD2}

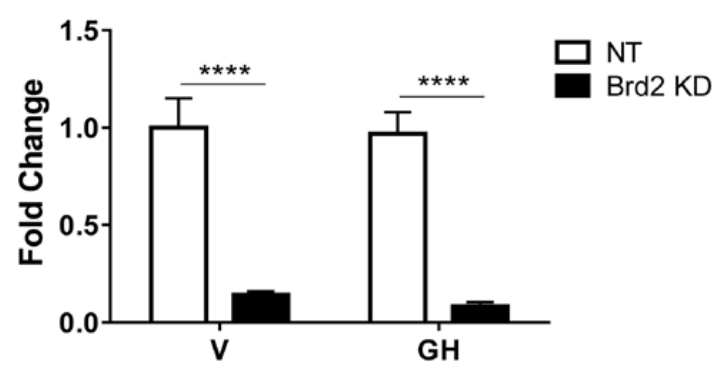

Figure 5. BRD2 knockdown (KD) downregulates growth hormone (GH)-induction of Cish and has no effect on Socs3 expression. Fully differentiated 3T3-L1 adipocytes were transfected with $50 \mathrm{nM}$ nontargeting (NT) siRNA or Brd2 siRNA. siRNA-containing media was removed 24 hours later and replaced with 10\% FBS. After 48 hours, adipocytes were transfected again with NT or Brd2 siRNA. siRNA-containing media was removed 24 hours later and replaced with 10\% FBS. After 24 hours, FBS was replaced by $1 \%$ CALF. The next day, cells were treated with vehicle (NaHCO3) or $5 \mathrm{nM}$ murine growth hormone $(\mathrm{GH})$ for 1 hour. (A) Cells were collected in RLT lysis buffer (Qiagen). Total RNA was isolated and subjected to RT-qPCR analysis. Brd2, Socs3 and Cish mRNA levels were measured. Nono was used as the reference gene. (B) Whole-cell extracts were collected in IP buffer, and $30 \mu \mathrm{g}$ of protein was used for gel electrophoresis. BRD2 and lamin B1 protein levels were detected by Western blot. Band intensities were quantified and fold change was calculated by dividing BRD2/lamin B2 and is represented below Western blot images. Data are presented as means \pm SE ( $n=3$ per treatment). This experiment was replicated 2 times in different batches of cells. Statistical significance was determined using two-way ANOVA. ${ }^{*} P<0.05, * * P<0.005, * * * P<0.001, * * * * P<0.0001$ for comparisons of NT versus Brd2 KD. Abbreviations: ANOVA, analysis of variance; CALF, bovine calf serum; FBS, fetal bovine serum; IP, immunoprecipitation; mRNA, messenger ribonucleic acid; RT-qPCR, reverse transcription-quantitative polymerase chain reaction; SE, standard error; siRNA, small interfering RNA. 

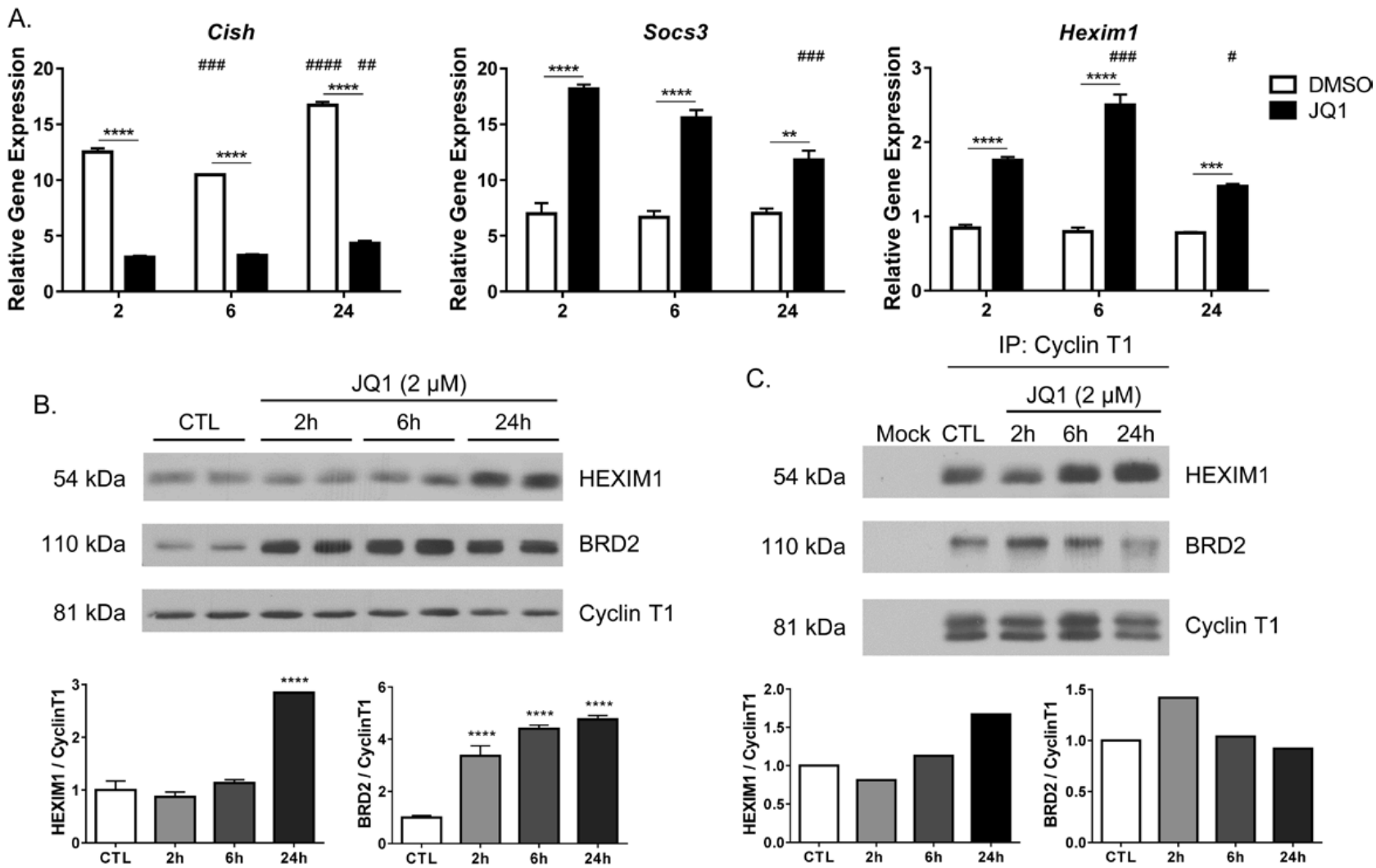

JQ1 $(2 \mu \mathrm{M})$
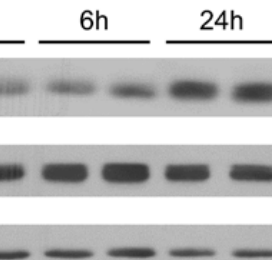

Cyclin T1

HEXIM1

BRD2
C.

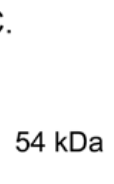

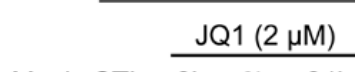

Mock CTL 2h $6 \mathrm{~h} \quad 24 \mathrm{~h}$

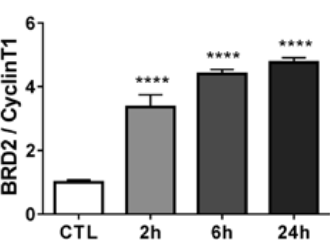

$110 \mathrm{kDa}$

$81 \mathrm{kDa}$

Figure 6. JQ1 transiently upregulates Hexim1 expression and decreases its interaction with cyclin T1. (A) Mature adipocytes were treated with DMSO or $2 \mu \mathrm{M}$ JQ1 for 2, 6, or 24 hours. Cish, Socs3, and Hexim1 gene expression levels were quantified by RT-qPCR. Nono was used as the reference gene. Samples were normalized to controls treated with DMSO (JQ1 vehicle) and $\mathrm{NaHCO}_{3}(\mathrm{GH}$ vehicle). Data from GH-treated samples are shown and presented as means \pm SE ( $n=3$ per treatment). Statistical significance was calculated using two-way ANOVA with the Bonferroni post hoc test. * denotes significance among DMSO and JQ1 treatment. \# denotes significance between 2 hours and the other time points.

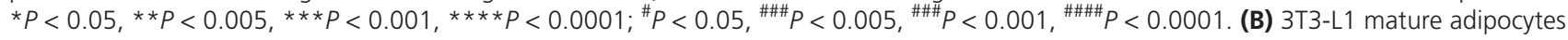
were treated with $2 \mu \mathrm{M}$ JQ1 for the indicated time points. Control (CTL) cells were untreated. Whole-cell extracts were collected in IP buffer, and $25 \mu \mathrm{g}$ of protein was used for gel electrophoresis. HEXIM1, BRD2, and cyclin T1 protein levels were detected by Western blot. (C) From the same experiment as B, $300 \mu \mathrm{g}$ of total protein was incubated with anti-cyclin T1 antibody overnight. Interacting HEXIM1 and BRD2 were detected by Western blot. The mock sample was composed of a pool of all samples without any antibody. For B and C, band intensities were quantified and cyclin T1 was used as a loading control to calculate fold change. Quantification graphs are shown below immunoblot images. Statistical significance was calculated using one-way ANOVA. * denotes significance between CTL and other treatments as described for A. Abbreviations: ANOVA, analysis of variance; DMSO, dimethyl sulfoxide; GH, growth hormone; RT-qPCR, reverse transcription-quantitative polymerase chain reaction; SE, standard error.

the Western blot images. For the 2-hour JQ1 treatment, the interaction between cyclin T1 and HEXIM1 was reduced, while interaction with BRD2 was increased. As the interaction with HEXIM1 increased over time, the interaction with BRD2 decreased. The highest increase in Socs3 expression occurs at 2 hours for JQ1 treatment, when the interaction between HEXIM1 and cyclin T1 is maximally inhibited. As HEXIM1 protein levels increase and P-TEFb complex is reassembled, the JQ1-induced increase in GH-activated Socs 3 expression decreases. These results confirmed that JQ1 has an offtarget effect on $\mathrm{P}-\mathrm{TEFb}$ availability in adipocytes.

\section{Discussion}

The transcription factor STAT5 has been extensively studied for its pathophysiological roles in cancer because its constitutive activation can lead to dysregulation of cell development and survival (40-43); it is also known to play an important role in fat cell development and function $(5,44,45)$. However, the exact molecular mechanisms by which STAT5 impacts cell fate and physiology are still poorly understood. The BET protein, BRD2, has been reported to be involved in STAT5-mediated transcription $(14,21)$. In our investigation, treatment of adipocytes with the BET inhibitor JQ1 produced divergent effects on 2 well-known STAT5 target genes. GH activation of Cish was reduced, while activation of Socs 3 was even greater in the presence of JQ1 (Fig. 2). 
ChIP was used to investigate the interaction of BET proteins, STAT5, and RNA Pol II with the Socs3 and Cish promoters, as well as the enrichment of RNA Pol II phosphorylated at Ser2 on Socs 3 and Cish coding regions. Changes in STAT5 binding were similar for both genes, indicating that this was not driving the divergent gene expression patterns (Figs 3B and 4B). Binding of both BRD2 and RNA Pol II to the Cish TSS was increased by GH and suppressed by the BET inhibitor JQ1 (Fig. 3C and E). We therefore hypothesized that BRD2, which is required for $\mathrm{GH}$-induced Cish transcription, was displaced from the TSS by JQ1, thus affecting RNA Pol II recruitment and consequently decreasing the ability of GH to induce Cish gene expression. Since BET proteins bind to acetylated histones, we also assessed histone $\mathrm{H} 3$ and $\mathrm{H} 4$ acetylation at the Socs 3 and Cish promoters and observed higher total acetylation levels at the Cish (Fig. 3G and $\mathrm{H}$ ) promoter compared with Socs3 (Fig. 4G and H). In addition, at basal conditions, GH increased acetylation at the Cish, but not the Socs3, TSS (transcription start site). These results support the hypothesis that $\mathrm{GH}$ induction of Cish gene expression is dependent on increased acetylation at the promoter to facilitate the recruitment of BRD2 to acetylated lysines at the Cish TSS. In the presence of JQ1, BRD2 binding is decreased and may consequently affect recruitment of the basal transcription machinery. This phenomenon has been similarly described for interleukin-3 activation of Cish in Ba/F3 cells (21). BRD2 involvement in GH activation of Cish was further confirmed by the observed reduction in Cish expression when BRD2 was knocked down using siRNA (Fig. 5A).

BRD2 was not recruited to the Socs 3 promoter by $\mathrm{GH}$, and BET inhibition had only minor effects on BRD2 binding to the Socs3 TSS (Fig. 4C), suggesting that BRD2 is not required for GH-regulated Socs3 expression. RNA Pol II recruitment to Socs3 was enhanced by JQ1 in basal conditions, but not in the presence of GH (Fig. 4E). On the other hand, enrichment of phosphorylated Pol II to the coding region of Socs3 was highly augmented by JQ1 (Fig. 4F). Since RNA Pol II phosphorylated at Ser2 is a well-known marker of elongation, we concluded that JQ1 increases Socs $3 \mathrm{mRNA}$ elongation. Although we did not directly measure the binding of the elongation factor P-TEFb to Cish or Socs 3 promoters, we know that RNA Pol II is primarily phosphorylated at Ser2 by P-TEFb in metazoans (46).

The upregulation of Socs3 by JQ1 was at first challenging to explain; however, extensive work done in the HIV field has shed light on how the inhibition of BET proteins could upregulate some genes. In fact, the phenomenon does not necessarily involve BET proteins but may instead constitute an off-target effect whereby JQ1 (18) and other BET inhibitors (47) induce the release of P-TEFb from its inhibitory complex. HEXIM1 acts to inhibit P-TEFb when they are both associated with 7SK small nuclear RNA (48). In our experiments, the decreased association between cyclin T1 (the regulatory subunit of P-TEFb) and HEXIM1 following JQ1 exposure coupled with the increased association between BRD2 and cyclin T1 (Fig. 6C), as well as the upregulation of HEXIM1 (Fig. 6B), support the hypothesis that JQ1 interferes with the binding of P-TEFb to its inhibitory complex. Disruption of the P-TEFb inhibitory complex increases P-TEFb availability and also activates cellular feedback mechanisms to upregulate HEXIM1 expression and reassemble the 7SK small nuclear RNA inhibitory complex $(18,49)$. Nonetheless, our findings do not exclude the possibility that the upregulation of Socs3 by JQ1 is not a direct effect on HEXIM1-P-TEFb interaction, and other modulators of mRNA elongation might be affected by BET inhibition.

Most studies have focused on BRD4 interaction with the P-TEFb complex via its cyclin T1 subunit. Our study confirms that BRD2 can also be present in a complex with cyclin T1. Further investigation will be required to determine whether this interaction is direct or mediated by other proteins. Among all BETs, only BRD4 possesses a C-terminal segment called P-TEFb interacting domain that can actively dissociate P-TEFb from HEXIM1. But depletion of that domain does not completely eliminate the association between BRD4 and cyclinT1 $(50,51)$, indicating that another region of BET proteins may facilitate this interaction.

Our results support the model shown in Fig. 7 where the upregulation of Socs3 by JQ1 is due to interference with the P-TEFb inhibitory complex. While more studies will be needed to fully explain the divergent transcriptional regulation of Socs 3 and Cish, our hypothetical model suggests that GH upregulation of Cish is dependent on BRD2 recruitment to the promoter and on recruitment of RNA Pol II and P-TEFb for transcript elongation. When JQ1 is present, it displaces BRD2 from the Cish promoter, thus decreasing its gene expression. GH upregulation of Socs3, on the other hand, is likely dependent on another factor. Since P-TEFb, when not bound to its inhibitory complex, is reported to associate with either BRD4 or the SEC $(19,52,53)$, we hypothesize that SEC is responsible for recruiting $\mathrm{P}-\mathrm{TEFb}$ to the Socs3 promoter. When JQ1 is present, it disrupts the interaction between P-TEFb and HEXIM1, allowing more $\mathrm{P}-\mathrm{TEFb}$ to be available for recruitment by SEC, which could further increase GH-induced Socs3 expression (Fig. 7). 

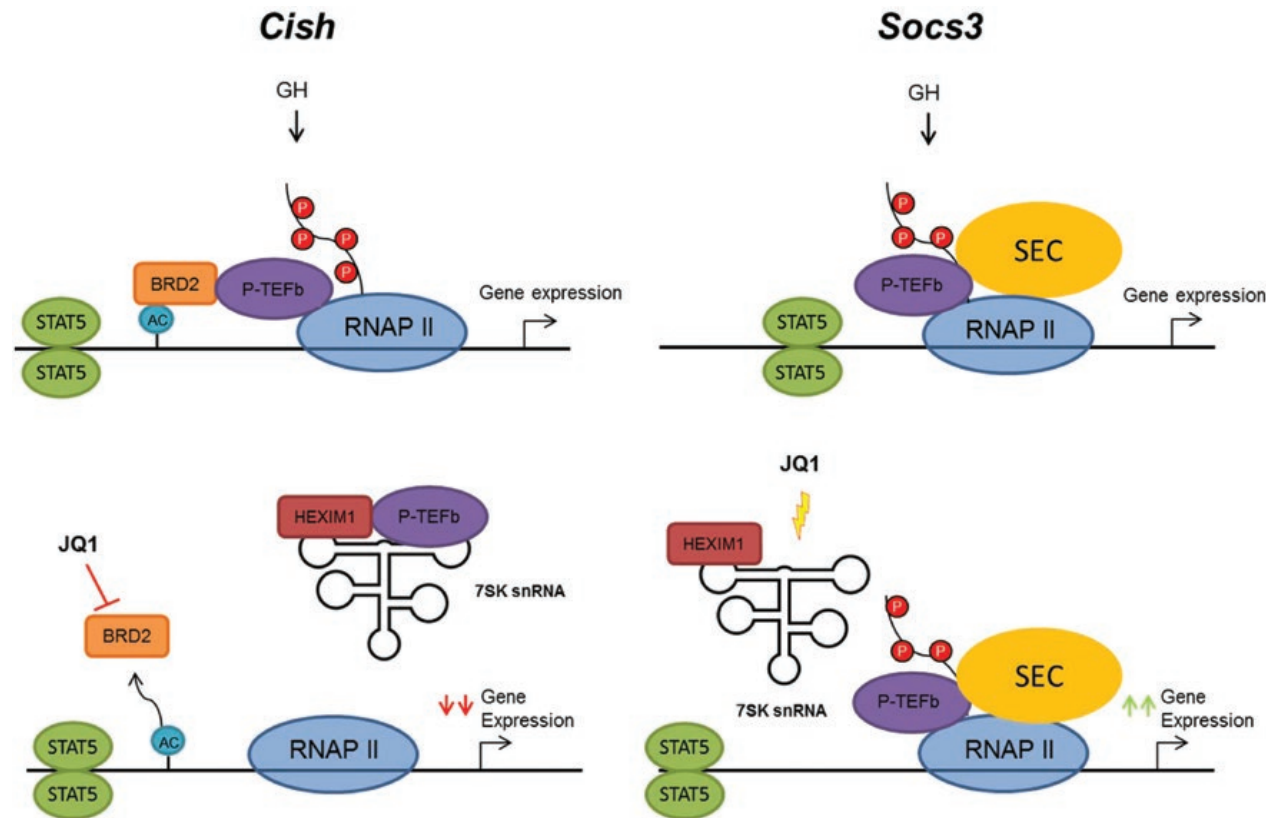

Figure 7. Model for potential differences between Socs3 and Cish gene expression. Under normal conditions, when 3T3-L1 adipocytes are stimulated with GH, the JAK-STAT signaling pathway is activated. STAT5 dimerizes and binds to STAT binding sites within the Cish and Socs3 promoters. For the Cish promoter, BRD2 is recruited by GH treatment. Then, BRD2 binds acetylated histones and recruits the positive elongation factor (P-TEFb), which is composed of 2 CDK9 and 2 cyclin T1 subunits. P-TEFb is responsible for the phosphorylation of Ser2 on the RNA Pol II C-terminal domain (CTD) to promote elongation. When the cells are pretreated with JQ1 before GH stimulation, BRD2 is displaced from chromatin. Therefore, P-TEFb is not recruited to Cish promoter. At the Socs3 promoter, GH treatment stimulates the recruitment of P-TEFb by another factor, possibly the super elongation complex (SEC). P-TEFb then phosphorylates RNA Pol II and productive elongation occurs. When the cells are pretreated with JQ1, in addition to release of BRD2 from chromatin, it also releases P-TEFb from its inhibitory complex. P-TEFb then becomes more available to promote Socs3 elongation. Abbreviations: GH, growth hormone; JAK, Janus Kinase; RNA Pol II, ribonucleic acid polymerase II; Ser2, serine 2; STAT, signal transducer and activator of transcription.

SOCS3 and CISH are members of the suppressors of the cytokine signaling family. All members of this family possess a conserved Src homology 2 domain and a C-terminal SOCS box. The Src homology 2 domain inhibits the signaling pathway via direct or competitive binding to phosphorylated intermediates, while the SOCS box can associate with ubiquitination machinery that targets proteins to the proteasome for degradation (54). Studies comparing SOCS proteins have revealed different mechanisms of inhibition among the family members. SOCS3 and SOCS1 possess a kinase inhibitory region (55) that is responsible for Janus kinase (JAK) inhibition. SOCS3 can also bind simultaneously to JAK and cytokine receptors, revealing higher levels of specificity $(56,57)$. On the other hand, CISH does not interact with JAKs. The binding of CISH to more distal tyrosine residues of the growth hormone receptor (GHR) leads to direct competition with STAT5 binding sites. Alternatively, CISH is involved in a proteasomedependent mechanism that may induce GHR internalization and destruction of GHR-JAK2-CISH complexes $(6,58)$.

Differences in the activation patterns of Socs 3 and Cish have been described $(34,37)$, and we have confirmed these differences in our studies. The fast and acute upregulation of Socs3, with activation peaking between 30 minutes and 1 hour and a return to basal levels in 2 hours, represents a classical feedback loop. Cish, however, is upregulated a little later and remains highly elevated even after 48 hours of GH stimulation. Therefore, despite similar protein structures and roles in inhibiting cytokine signaling pathways, differences in gene activation patterns and divergent responses to BET inhibition demonstrate that Socs 3 and Cish may have different functions, at least in the context of cultured murine adipocytes.

The role of BET proteins in epigenetics and gene regulation has been an intensive area of research. Preclinical studies have shown that the BET inhibitor OTX015 affects JAK/STAT signaling and can increase Hexim1 expression (59), suggesting an off-target effect on P-TEFb regulation. Moreover, OTX015, is now in a phase $1 \mathrm{~b}$ clinical trial in patients with selected advanced solid tumors (60). The results of our study also suggest an off-target effect of JQ1 on P-TEFb regulation that influences STAT5 target gene expression in adipocytes. The possibility that BET inhibitors affect the availability of $\mathrm{P}-\mathrm{TEFb}$, the primary regulator of mRNA elongation, has potentially broad biological consequences and should be taken into consideration when these drugs are considered for therapeutic use. Furthermore, the fact that 2 
very similar genes, Cish and Socs3, utilize different transcriptional machinery, resulting in such opposite regulation in the presence of JQ1, demonstrates how unique and complex the regulation of GH-induced gene expression is and may have important implications in the feedback regulation of JAK/STAT signaling in adipocytes.

\section{Acknowledgments}

We thank Hardy Hang for technical support and Anik Boudreau for manuscript editing.

Financial Support: This project was supported by the R01DK052968 grant to J.M.S. from the National Institutes of Diabetes and Digestive and Kidney Diseases. This project used a genomics core facility supported in part by COBRE (NIH8 1P30GM118430-01) and NORC (NIH 1P30-DK072476) center grants from the National Institutes of Health. P.M.dS. had a scholarship from the Brazilian National Council for Scientific and Technological Development (CNPq).

Author Contributions: P.M.dS., A.J.R., and J.M.S. designed the experiments. P.M.dS. conducted the experiments. P.M.dS., A.J.R., and J.M.S. analyzed the results. P.M.dS. wrote the majority of the manuscript; A.J.R. and J.M.S. edited the paper.

\section{Additional Information}

Correspondence: Jacqueline Stephens, Adipocyte Biology Laboratory, Pennington Biomedical Research Center, Louisiana State University, Baton Rouge, Louisiana 70803. E-mail: jsteph1@lsu.edu.

Disclosure Summary: The authors have nothing to disclose.

\section{References}

1. Darnell JE Jr. STATs and gene regulation. Science. 1997;277(5332):1630-1635.

2. Trengove MC, Ward AC. SOCS proteins in development and disease. Am J Clin Exp Immunol. 2013;2(1):1-29.

3. Zvonic S, Story DJ, Stephens JM, Mynatt RL. Growth hormone, but not insulin, activates STAT5 proteins in adipocytes in vitro and in vivo. Biochem Biophys Res Commun. 2003;302(2):359-362.

4. Fleenor D, Arumugam R, Freemark M. Growth hormone and prolactin receptors in adipogenesis: STAT-5 activation, suppressors of cytokine signaling, and regulation of insulin-like growth factor I. Horm Res. 2006;66(3):101-110.

5. Floyd ZE, Stephens JM. STAT5A promotes adipogenesis in nonprecursor cells and associates with the glucocorticoid receptor during adipocyte differentiation. Diabetes. 2003;52(2):308-314.

6. Ram PA, Waxman DJ. SOCS/CIS protein inhibition of growth hormone-stimulated STAT5 signaling by multiple mechanisms. $J$ Biol Chem. 1999;274(50):35553-35561.

7. Woelfle J, Rotwein P. In vivo regulation of growth hormonestimulated gene transcription by STAT5b. Am J Physiol Endocrinol Metab. 2004;286(3):E393-E401.

8. Hochberg I, Tran QT, Barkan AL, Saltiel AR, Chandler WF, Bridges D. Gene expression signature in adipose tissue of acromegaly patients. Plos One. 2015;10(6):e0129359.

9. Lin G, LaPensee CR, Qin ZS, Schwartz J. Reciprocal occupancy of BCL6 and STAT5 on growth hormone target genes: contrasting transcriptional outcomes and promoter-specific roles of p300 and HDAC3. Mol Cell Endocrinol. 2014;395(1-2):19-31.

10. Yang Z, Yik JH, Chen R, et al. Recruitment of P-TEFb for stimulation of transcriptional elongation by the bromodomain protein Brd4. Mol Cell. 2005;19(4):535-545.

11. Peterlin BM, Price DH. Controlling the elongation phase of transcription with P-TEFb. Mol Cell. 2006;23(3):297-305.

12. Dawson MA, Prinjha RK, Dittmann A, et al. Inhibition of BET recruitment to chromatin as an effective treatment for MLL-fusion leukaemia. Nature. 2011;478(7370):529-533.

13. Wyce A, Degenhardt Y, Bai Y, et al. Inhibition of BET bromodomain proteins as a therapeutic approach in prostate cancer. Oncotarget. 2013;4(12):2419-2429.

14. Liu S, Walker SR, Nelson EA, et al. Targeting STAT5 in hematologic malignancies through inhibition of the bromodomain and extra-terminal (BET) bromodomain protein BRD2. Mol Cancer Ther. 2014;13(5):1194-1205.

15. Belkina AC, Nikolajczyk BS, Denis GV. BET protein function is required for inflammation: Brd2 genetic disruption and BET inhibitor JQ1 impair mouse macrophage inflammatory responses. $J$ Immunol. 2013;190(7):3670-3678.

16. Meng S, Zhang L, Tang Y, et al. BET Inhibitor JQ1 blocks inflammation and bone destruction. J Dent Res. 2014;93(7): 657-662.

17. Mancebo HS, Lee G, Flygare J, et al. P-TEFb kinase is required for HIV Tat transcriptional activation in vivo and in vitro. Genes Dev. 1997;11(20):2633-2644.

18. Bartholomeeusen K, Xiang Y, Fujinaga K, Peterlin BM. Bromodomain and extra-terminal (BET) bromodomain inhibition activate transcription via transient release of positive transcription elongation factor $\mathrm{b}(\mathrm{P}-\mathrm{TEFb})$ from $7 \mathrm{SK}$ small nuclear ribonucleoprotein *. J Biol Chem. 2012;287(43):36609-16.

19. Li Z, Guo J, Wu Y, Zhou Q. The BET bromodomain inhibitor JQ1 activates HIV latency through antagonizing Brd4 inhibition of Tat-transactivation. Nucleic Acids Res. 2013;41(1):277-287.

20. Bartholomeeusen K, Fujinaga K, Xiang Y, Peterlin BM. Histone deacetylase inhibitors (HDACis) that release the positive transcription elongation factor $\mathrm{b}(\mathrm{P}-\mathrm{TEFb})$ from its inhibitory complex also activate HIV transcription. $\mathrm{J}$ Biol Chem. 2013;288(20):14400-14407.

21. Pinz S, Unser S, Buob D, Fischer P, Jobst B, Rascle A. Deacetylase inhibitors repress STAT5-mediated transcription by interfering with bromodomain and extra-terminal (BET) protein function. Nucleic Acids Res. 2015;43(7):3524-3545.

22. RRID:AB_10835146, https://antibodyregistry.org/search. php?q=AB_10835146.

23. RRID:AB_2650517, https://antibodyregistry.org/search?q=RRID: AB_2650517.

24. RRID:AB_1031062, https://antibodyregistry.org/search?q=RRID: AB_1031062.

25. RRID:AB_304749, https://antibodyregistry.org/search?q=RRID: AB_304749.

26. RRID:AB_2814653, https://antibodyregistry.org/search?q=RRID: AB_2814653.

27. RRID:AB_2233058, https://antibodyregistry.org/search?q=RRID: AB_2233058.

28. RRID:AB_778107, https://antibodyregistry.org/search?q=RRID: AB_778107.

29. RRID:AB_2610136, https://antibodyregistry.org/search?q=RRID: AB_2610136.

30. RRID:AB_2115283, https://antibodyregistry.org/search.php? $\mathrm{q}=\mathrm{AB} \_2115283$.

31. RRID:AB_310270, https://antibodyregistry.org/search.php? $\mathrm{q}=\mathrm{AB} \_310270$.

32. RRID:AB_1163497, https://antibodyregistry.org/search?q=RRID: AB_1163497.

33. Zhu BM, Kang K, Yu JH, et al. Genome-wide analyses reveal the extent of opportunistic STAT5 binding that does not yield 
transcriptional activation of neighboring genes. Nucleic Acids Res. 2012;40(10):4461-4472.

34. Tollet-Egnell P, Flores-Morales A, Stavréus-Evers A, Sahlin L, Norstedt G. Growth hormone regulation of SOCS-2, SOCS3 , and CIS messenger ribonucleic acid expression in the rat. Endocrinology. 1999;140(8):3693-3704.

35. Martinez CS, Piazza VG, Díaz ME, et al. GH/STAT5 signaling during the growth period in livers of mice overexpressing GH. $J$ Mol Endocrinol. 2015;54(2):171-184.

36. Barclay JL, Anderson ST, Waters MJ, Curlewis JD. Regulation of suppressor of cytokine signaling 3 (SOC3) by growth hormone in pro-B cells. Mol Endocrinol. 2007;21(10):2503-2515.

37. Adams TE, Hansen JA, Starr R, Nicola NA, Hilton DJ, Billestrup N. Growth hormone preferentially induces the rapid, transient expression of SOCS-3, a novel inhibitor of cytokine receptor signaling. J Biol Chem. 1998;273(3):1285-1287.

38. Filippakopoulos P, Qi J, Picaud S, et al. Selective inhibition of BET bromodomains. Nature. 2010;468(7327):1067-1073.

39. Trabucco SE, Gerstein RM, Evens AM, et al. Inhibition of bromodomain proteins for the treatment of human diffuse large B-cell lymphoma. Clin Cancer Res. 2015;21(1):113-122.

40. Koppikar P, Lui VW, Man D, et al. Constitutive activation of signal transducer and activator of transcription 5 contributes to tumor growth, epithelial-mesenchymal transition, and resistance to epidermal growth factor receptor targeting. Clin Cancer Res. 2008;14(23):7682-7690.

41. Li H, Ahonen TJ, Alanen K, et al. Activation of signal transducer and activator of transcription 5 in human prostate cancer is associated with high histological grade. Cancer Res. 2004;64(14):4774-4782.

42. Nevalainen MT, Xie J, Torhorst J, et al. Signal transducer and activator of transcription-5 activation and breast cancer prognosis. J Clin Oncol. 2004;22(11):2053-2060.

43. Moriggl R, Sexl V, Kenner L, et al. Stat5 tetramer formation is associated with leukemogenesis. Cancer Cell. 2005;7(1):87-99.

44. Stephens JM, Morrison RF, Pilch PF. The expression and regulation of STATs during 3T3-L1 adipocyte differentiation. J Biol Chem. 1996;271(18):10441-10444.

45. Teglund S, McKay C, Schuetz E, et al. Stat5a and Stat 5 b proteins have essential and nonessential, or redundant, roles in cytokine responses. Cell. 1998;93(5):841-850.

46. Bowman EA, Kelly WG. RNA polymerase II transcription elongation and Pol II CTD Ser2 phosphorylation: a tail of two kinases. Nucleus. 2014;5(3):224-236.
47. Lu P, Qu X, Shen Y, et al. The BET inhibitor OTX015 reactivates latent HIV-1 through P-TEFb. Sci Rep. 2016;6:24100.

48. Michels AA, Fraldi A, Li Q, et al. Binding of the 7SK snRNA turns the HEXIM1 protein into a P-TEFb (CDK9/cyclin T) inhibitor. Embo J. 2004;23(13):2608-2619.

49. He N, Pezda AC, Zhou Q. Modulation of a P-TEFb functional equilibrium for the global control of cell growth and differentiation. Mol Cell Biol. 2006;26(19):7068-7076.

50. Schröder S, Cho S, Zeng L, et al. Two-pronged binding with bromodomain-containing protein 4 liberates positive transcription elongation factor $\mathrm{b}$ from inactive ribonucleoprotein complexes. J Biol Chem. 2012;287(2):1090-1099.

51. Taniguchi Y. The bromodomain and extra-terminal domain (BET) family: functional anatomy of BET paralogous proteins. Int J Mol Sci. 2016;17(11):pii: E1849.

52. Smith E, Lin C, Shilatifard A. The super elongation complex (SEC) and MLL in development and disease. Genes Dev. 2011;25(7):661-672.

53. Zhou Q, Li T, Price DH. RNA polymerase II elongation control. Annu Rev Biochem. 2012;81:119-143.

54. Babon JJ, Sabo JK, Zhang JG, Nicola NA, Norton RS. The SOCS box encodes a hierarchy of affinities for Cullin5: implications for ubiquitin ligase formation and cytokine signalling suppression. $J$ Mol Biol. 2009;387(1):162-174.

55. Sasaki A, Yasukawa H, Suzuki A, et al. Cytokine-inducible SH2 protein-3 (CIS3/SOCS3) inhibits Janus tyrosine kinase by binding through the N-terminal kinase inhibitory region as well as $\mathrm{SH} 2$ domain. Genes Cells. 1999;4(6):339-351.

56. Babon JJ, Kershaw NJ, Murphy JM, et al. Suppression of cytokine signaling by SOCS3: characterization of the mode of inhibition and the basis of its specificity. Immunity. 2012;36(2):239-250.

57. Kershaw NJ, Murphy JM, Liau NP, et al. SOCS3 binds specific receptor-JAK complexes to control cytokine signaling by direct kinase inhibition. Nat Struct Mol Biol. 2013;20(4):469-476.

58. Landsman T, Waxman DJ. Role of the cytokine-induced $\mathrm{SH} 2$ domain-containing protein CIS in growth hormone receptor internalization. J Biol Chem. 2005;280(45):37471-37480.

59. Coudé MM, Braun T, Berrou J, et al. BET inhibitor OTX015 targets BRD2 and BRD4 and decreases c-MYC in acute leukemia cells. Oncotarget. 2015;6(19):17698-17712.

60. Lewin J, Soria JC, Stathis A, et al. Phase Ib trial with birabresib, a small-molecule inhibitor of bromodomain and extraterminal proteins, in patients with selected advanced solid tumors. J Clin Oncol. 2018;36(30):3007-3014. 